\title{
Recent results on Bose-Einstein correlations by the PHENIX Experiment
}

\author{
Vicki Greene* for the PHENIX Collaboration \\ Vanderbilt University \\ E-mail: greene@vanderbilt.edu
}

\begin{abstract}
We present the measurement of charged pion two-particle correlation functions in $\sqrt{s_{N N}}=39$, 62 and $200 \mathrm{GeV} \mathrm{Au+Au}$ collisions. The measured correlation functions for all collision energies, centralities and average transverse masses can be statistically well described by Bose-Einstein correlation functions from Lévy-shaped source distributions. We extract the correlation strength parameter $\lambda$, Lévy index $\alpha$ and Lévy scale parameter $R$ as functions of average pair transverse mass. We observe that the Lévy index $\alpha$ differs from the Gaussian case of $\alpha=2$ and also from the conjectured value at the critical point. The correlation strength $\lambda$ shows a decrease for small transverse masses, which may be in conjunction with an increased number of decay pions in the given kinematic domain. The Lévy scale $R$ shows a hydrodynamic type of scaling behavior. Furthermore, the appearance of a new scaling variable $\widehat{R}$ is observed.
\end{abstract}

CPOD2017: Critical Point and Onset of Deconfinement

August 7-11, 2017

The Charles B. Wang Center, Stony Brook University, Stony Brook, NY

${ }^{*}$ Speaker. 


\section{Introduction}

In high energy physics, measurements of quantum statistical (Bose-Einstein, HBT) correlations has an important role because it can be used to gain insight into the space-time geometry of the particle emitting source. This sub-field of high energy physics, often called femtoscopy [1], includes methods which allow the measurements of femtometer scale lengths. It is based on the intensity correlation measurements of R. Hanbury Brown and R. Q. Twiss (HBT) [2], and the interpretation of these by R. Glauber [3-5]. These quantum statistical correlations were also observed and explained independently by G. Goldhaber and collaborators in proton-antiproton collisions $[6,7]$. They based their explanations on Bose-Einstein symmetrization of the pair wave function, and this result is now widely used in high energy heavy ion physics as well.

If we define $S(x, k)$, the probability density of creating a particle of a given species at $x$ with momentum $k$, then the momentum correlation function will be related to the Fourier transform of $S$ as:

$$
C_{2}^{(0)}(Q, K)=1+\left|\frac{\widetilde{S}(Q, K)}{\widetilde{S}(0, K)}\right|^{2}, \text { where } \quad \widetilde{S}(Q, k)=\int S(x, k) \exp (i Q x / \hbar) d x
$$

and $Q=p_{1}-p_{2}$ is the momentum difference, $K=\left(p_{1}+p_{2}\right) / 2$ is the average momentum of the pair, and the superscript $(0)$ denotes that in this simple case, we did not take into account any interaction of the given particles (besides quantum statistics). In this result, $Q$ is usually taken as the "main" variable of the correlation function, and correlation functions are measured in various ranges of $K$. Because of the central limit theorem, Gaussian-type sources may appear, which lead to Gaussian correlation functions as

$$
S(x, k) \propto \exp \left(-\frac{r^{2}}{R(k)^{2}}\right) \Leftrightarrow C_{2}^{(0)}(Q, K)-1 \propto \exp \left(-\frac{Q^{2} R(K)^{2}}{2 \hbar^{2}}\right) .
$$

These $R(K)$ radii are then called the Gaussian HBT radii, which describe the source size. Hydrodynamics suggests $[8,9]$, that because of the expanding medium in relativistic heavy ion collisions, these radii depend on the transverse momentum $m_{T}=\sqrt{m^{2}+K_{T}^{2}}$ as $R^{-2} \propto a+b m_{T}$, and this relation has been confirmed in various collision systems at various energies [10,11]. For more details (also for the discussion about when the above, unity plus positive definite type of correlation functions appear), see Ref. [12].

In the last decade, it has been seen that in heavy ion collisions, the shape of Bose-Einstein correlations is indeed Gaussian $[13,14]$, but has a power-law tail. This may be connected to the non-applicability of the central limit theorem, and the appearance of anomalous diffusion, resulting in a Lévy type of distributions [12,15-17]. These can be expressed as

$$
\mathscr{L}(\alpha, R, r)=\frac{1}{(2 \pi)^{3}} \int d^{3} q e^{i q r} e^{-\frac{1}{2}|q R|^{\alpha}},
$$

where $R$ is the Lévy scale and $\alpha$ is Lévy index. For $\alpha=2$, one obtains a Gaussian, while for $\alpha=1$, a Cauchy distribution, and there are no simple analytical expressions for other $\alpha$ values. It is important to note that Lévy distributions lead to power-law tails, with an exponent (in 3 dimensions) of $-3-\alpha$. The spatial correlations of a system near the critical point are characterized also by a 
power-law of $r^{-3-\eta}$. Hence the $\alpha$ exponent of the Lévy source function is related to the familiar critical exponent $\eta$ of a system at a second order phase transition [12,18]. This critical exponent, and hence also the Lévy exponent, is conjectured to be 0.5 or even lower, based on the fact that the QCD quark-hadron transition at the critical point belongs to the universality class of the 3D Ising model, possibly with random external fields [18-22]. Hence the measurement of the Lévy exponent may also contribute to the search for the QCD critical point.

It is also important to note that in the measurement of Bose-Einstein correlations, while Eq. (1.1) would lead to $C_{2}^{(0)}(Q=0, K)=2$, usually a lower value is observed, i.e.

$$
\lim _{Q \rightarrow 0} C_{2}^{(0)}(Q, K)=1+\lambda(K)<2
$$

The average momentum dependent intercept parameter $\lambda(K)$ is then the measured strength of correlation functions. This may be understood and interpreted in the frawework of the core-halo picture [23,24], where the source has two components: a core of the primordial pions (and the decay products of very short lived resonances, with lifetimes $\lesssim 10 \mathrm{fm} / c$ ), and a halo of the decay pions (coming from long lived resonances, with lifetimes $>50 \mathrm{fm} / \mathrm{c}$ ). This yields a two-component correlation function, and the narrow component can usually not be resolved (is below a minimal resolvable momentum difference). This leads to the following expression for the observed correlation strength:

$$
\lambda(K)=\left(\frac{N_{\text {core }}(K)}{N_{\text {core }}(K)+N_{\text {halo }}(K)}\right)^{2}
$$

where $N_{\text {core }}$ is the number of "core" pions, while $N_{\text {halo }}$ is the number of "halo" pions (both at a given $K)$. See more details in Ref [12]. Hence $\lambda(K)$ is related to the yield of resonances at the given momentum. It turns out $[25,26]$, that the $\eta^{\prime}$ in-medium mass is expected to be decreased for $U_{A}(1)$ symmetry restoration, which may lead to a decrease of $\lambda$ at low momenta - see more details in Refs. [12,27].

Let us furthermore note, that usually charged hadrons are measured, for which the above simple picture leading to $C_{2}^{(0)}$ is not sufficient. In this case, we have to take into account the electromagnetic and strong interactions. For identical charged pions, the Coulomb repulsion is the most important. This can be taken into account by using the Coulomb pair wave function as detailed in Ref. [12]. This leads to a "Coulomb-correction" $K_{2}(Q, K)$, which depends on the assumed source shape through the source parameters' $K$-dependence. With this, the measured correlation function can be described as

$$
C_{2}^{\text {measured }}(Q, K)=K_{2}(Q, K) C_{2}^{(0)}(Q, K)
$$

\section{Results from the $\sqrt{s_{N N}}=200 \mathrm{GeV} \mathbf{A u}+\mathbf{A u}$ 0-30\% centrality analysis}

We analyzed $\sim 7$ billion minimum bias $\mathrm{Au}+\mathrm{Au}$ events at $\sqrt{s_{N N}}=200 \mathrm{GeV}$ recorded by PHENIX during the 2010 running period. We selected $\sim 2.2$ billon events of $0-30 \%$ centrality, fulfilling basic event selection criteria. We measured two-pion correlation functions for $\pi^{+} \pi^{+}$and $\pi^{-} \pi^{-}$ 


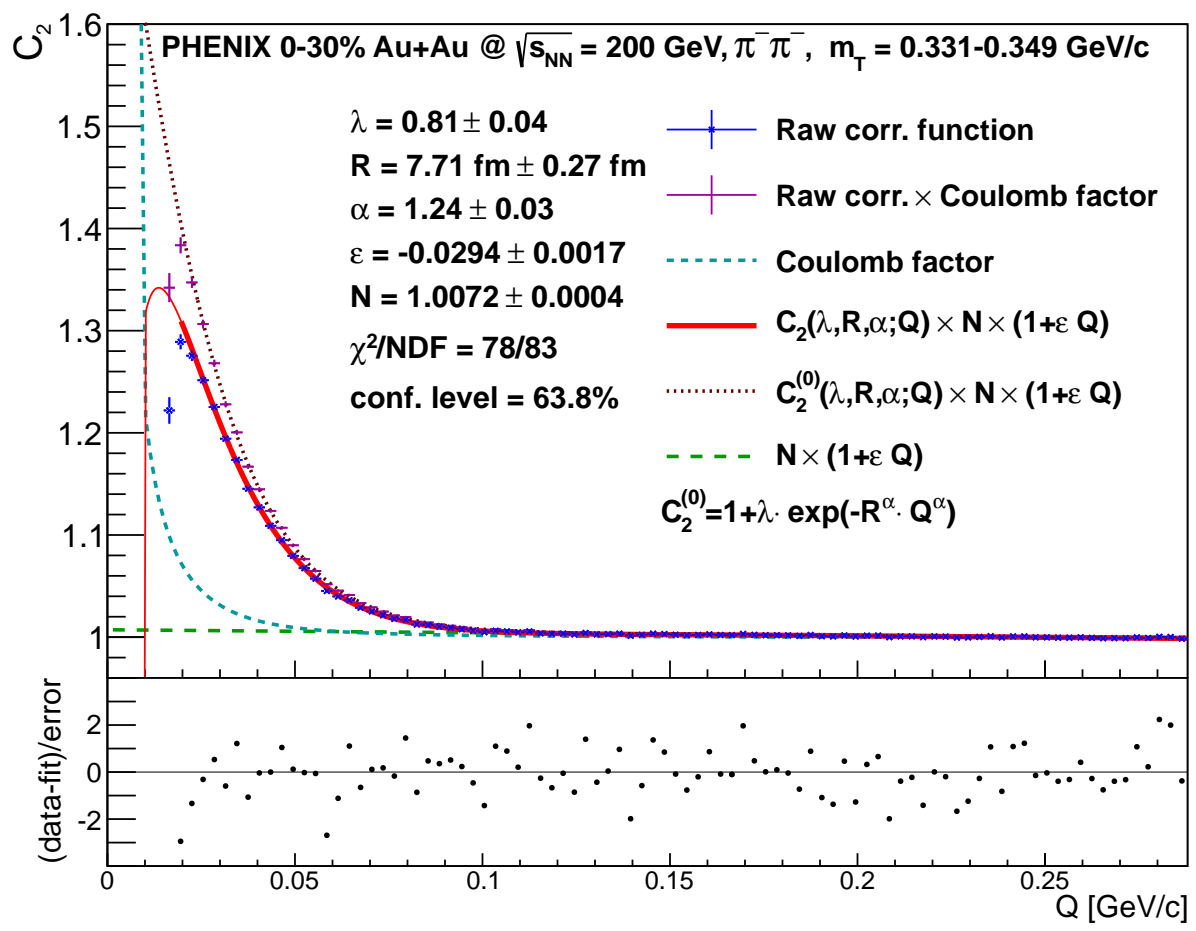

Figure 1: Example fit of a Bose-Einstein correlation function of $\pi^{+} \pi^{+}$pairs with average $p_{T}$ between 0.2 and $0.22 \mathrm{GeV} / \mathrm{c}$ measured in the longitudinal co-moving frame. In this analysis we measured and fit correlation functions in $31 m_{T}$ bins, for ++ and -- pairs separately.

pairs in $31 m_{T}$ bins (ranging from 228 to $871 \mathrm{MeV} / \mathrm{c}$ ). We obtained the correlation functions as

$$
C_{2}(Q, K)=\frac{A(Q, K)}{B(Q, K)} \cdot \frac{\int_{Q_{\min }}^{Q_{\max }} B(q, K) d q}{\int_{Q_{\min }}^{Q_{\max }} A(q, K) d q},
$$

where the integral is performed over a range $\left[Q_{\min }, Q_{\max }\right]$ where the correlation function is not expected to exhibit quantum statistical features. The analysis procedure and cuts are described in Refs. [12,28]. We fit these measured correlation functions with an iterative fit procedure (also described in Refs. [12] in detail), where the fit function was

$$
N\left(1+\lambda \exp \left[(Q R)^{\alpha}\right]\right)(1+\varepsilon k) K_{2}\left(\lambda_{0}, R_{0}, \alpha_{0} ; Q\right),
$$

where the $\lambda, R$ and $\alpha$ values thus obtained are plugged back into $K_{2}$, until their difference from $\lambda_{0}$, $R_{0}$ and $\alpha_{0}$ is less than $1 \%$ in squared sum. This type of iteration converged in 2-3 rounds usually, resulting in properly converged fits, accurate error matrix and calculable Minos uncertainties. An example fit is shown in Fig. 1, where the final fit function may be written as

$$
C_{2}(\lambda, R, \alpha ; Q)=N\left(1+\lambda \exp \left[(Q R)^{\alpha}\right]\right)(1+\varepsilon k) K_{2}(\lambda, R, \alpha ; Q) .
$$

The $m_{T}$ dependence of the resulting fit parameters is shown in Fig. 2. We observe that $\alpha$ is constant within systematic uncertainties, and takes an average value of 1.207, far from the Gaussian 

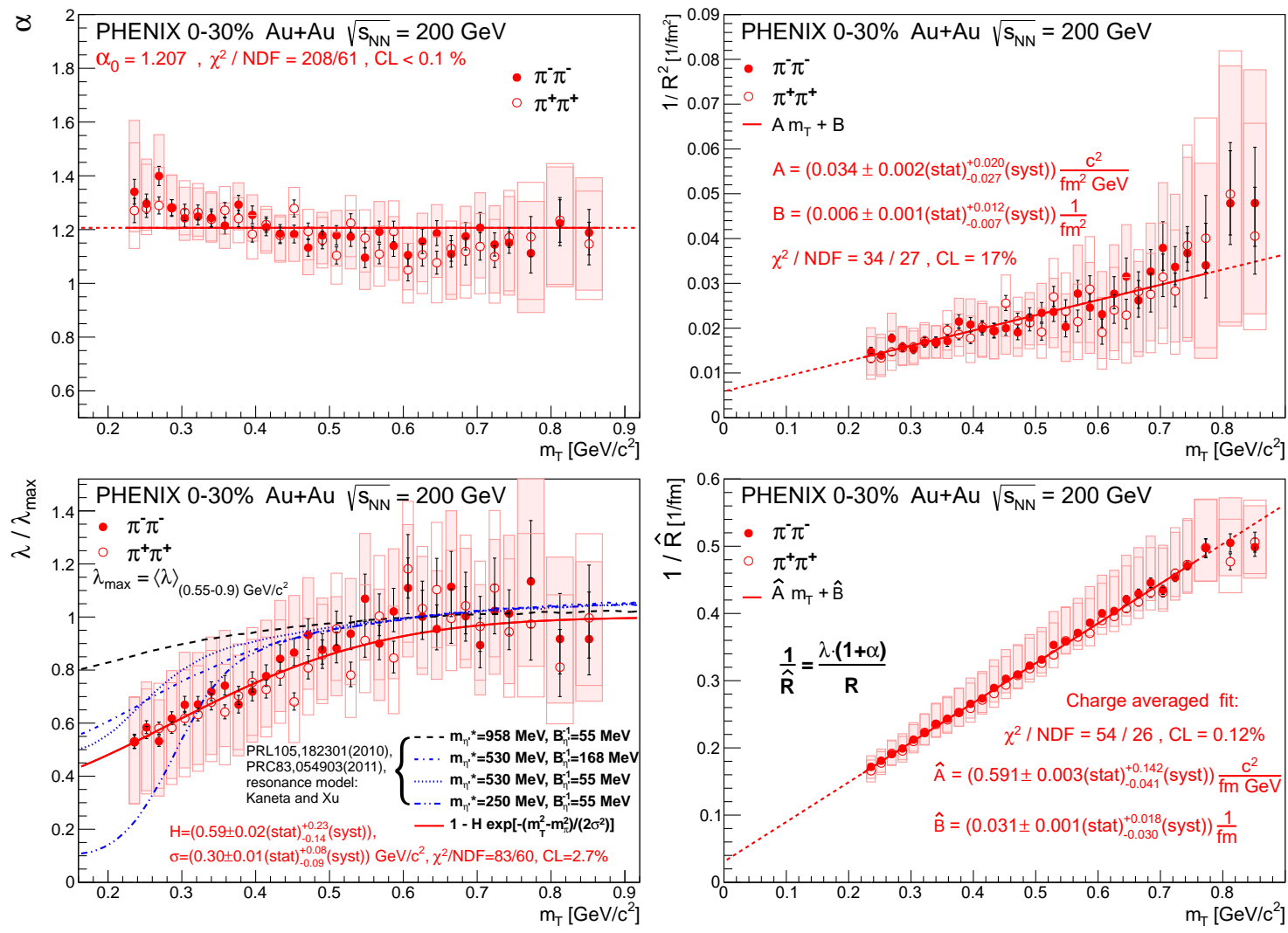

Figure 2: Fit parameters versus average $m_{T}$ of the pair with statistical and symmetric systematic uncertainties shown as bars and boxes, respectively.

assumption $(\alpha=2)$ or the conjectured $\alpha=0.5$ value at the critical point. The hydrodynamic prediction of $1 / R^{2} \simeq a+b m_{T}$ is also confirmed, despite the appearance of non-hydrodynamic power-law tails. The correlation function strength $\lambda$ is shown after a normalization by $\lambda_{\max }=$ $\langle\lambda\rangle_{m_{T}=0.5-0.7 \mathrm{GeV} / c^{2}}$, indicating a decrease at small $m_{T}$. This may be explained by the increase of the halo component at low $m_{T}$. This observation is not incompatible with predictions based on a reduced in-medium $\eta^{\prime}$ mass. We also show the observation of a new, empirically found scaling parameter $\widehat{R}=R /(\lambda(1+\alpha))$, showing a clear linear scaling with $m_{T}$.

\section{Collision energy and centrality dependence}

We performed a similar analysis as described in the previous subsection, for different centralities and center of mass energies, from $\sqrt{s_{N N}}=39 \mathrm{GeV}$ to $200 \mathrm{GeV}$ [29]. At $200 \mathrm{GeV}$, we defined six centrality classes from $0-10 \%$ to $50-60 \%$, at $62 \mathrm{GeV}$ four classes (from $0-10 \%$ to $30-40 \%$ ) and two classes at $39 \mathrm{GeV}(0-20 \%$ and $20-40 \%)$. After performing all the fits to all the measured correlation functions, we extracted the $m_{T}$-dependence of the fit parameters. For the $200 \mathrm{GeV}$ analysis, we used $18 m_{T}$ bins, 8 bins at $62 \mathrm{GeV}$ and 6 bins at $39 \mathrm{GeV}$. The extracted fit parameters (as a function of $m_{T}$ ) are shown in Figs. 3-6. We observe that $\alpha$ values (shown in Fig. 3) are in the 1-1.6 range, showing no clear $m_{T}$ dependence. As a function of centrality, non-monotonic 

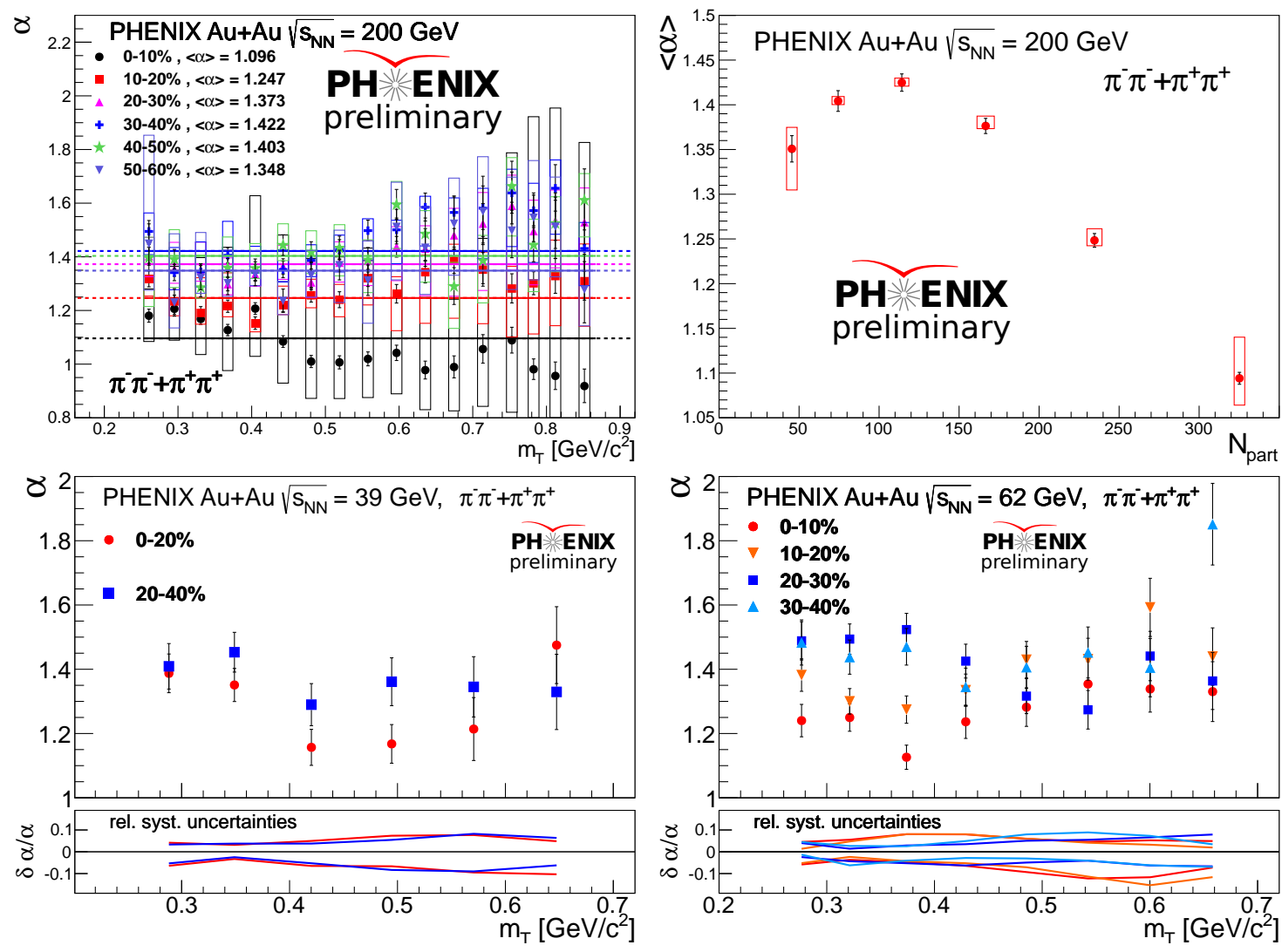

Figure 3: The fit parameter $\alpha$, for various centralities in $\sqrt{s_{N N}}=200 \mathrm{GeV} \mathrm{Au}+\mathrm{Au}$ collisions (top panels, free $\alpha$ on the left, fixed $\alpha$ fits on the right plot), and for lower collision energies (bottom panels). In the top panels, statistical and systematic uncertainties are indicated as bars and labels (respectively), while a separate subpanel is included for the systematic uncertainties in the lower plots.

behavior is observed with the highest $\alpha$ values around 30-40\%. No significant center of mass energy dependence is observed. For the $200 \mathrm{GeV}$ case, fits with fixed $\alpha$ (based on the average value indicated in Fig. 3) were also performed.The Lévy scales are shown in Fig. 4, plotted as $1 / R^{2}$ at $200 \mathrm{GeV}$. At $200 \mathrm{GeV}$, and especially for the fixed $\alpha$ case, the $1 / R^{2} \propto a+b m_{T}$ type of behavior is clearly observed. At the lower energies, statistical precision just allows us to observe the decreasing trend with $m_{T}$. Correlation strength $\lambda$ is shown in Fig. 5. The low- $m_{T}$ decrease is observable at all energies and centralities, most clearly for the fixed $\alpha$ fits at $200 \mathrm{GeV}$. The new scaling variable $\widehat{R}$ is shown in Fig. 6, clearly exhibiting an $1 / \widehat{R} \propto m_{T}$ trend for all energies and centralities, independently of whether $\alpha$ is fixed.

\section{References}

[1] R. Lednicky, nucl-th/0112011.

[2] R. Hanbury Brown and R. Q. Twiss, Nature 178, 1046 (1956).

[3] R. J. Glauber, Phys. Rev. Lett. 10, 84 (1963). 

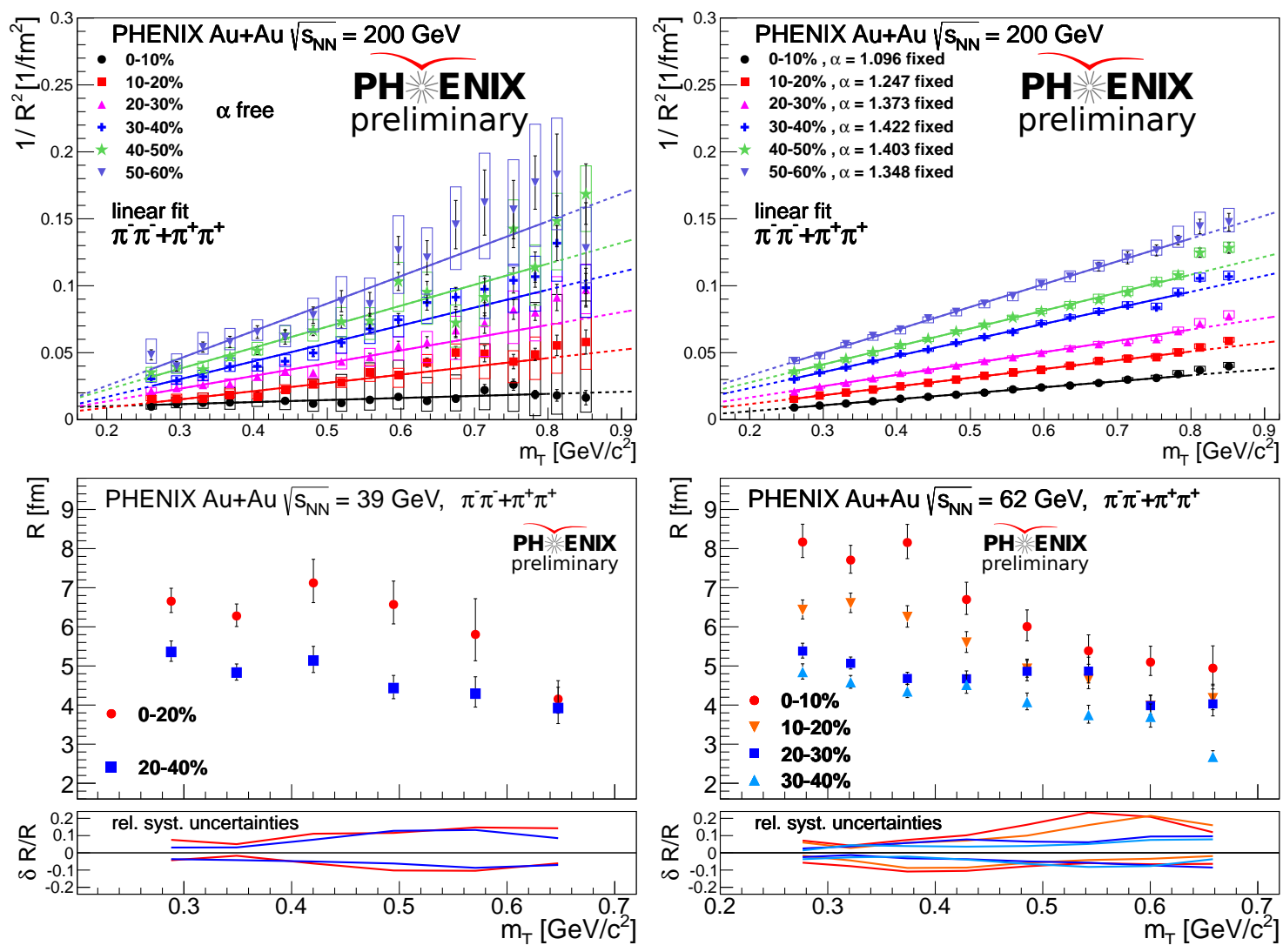

Figure 4: The fit parameter $R$, for various centralities in $\sqrt{s_{N N}}=200 \mathrm{GeV} \mathrm{Au}+\mathrm{Au}$ collisions (top panels, free $\alpha$ on the left, fixed $\alpha$ fits on the right plot), and for lower collision energies (bottom panels). Statistical and systematic uncertainties are indicated similarly to Fig. 3.

[4] R. J. Glauber, Rev. Mod. Phys. 78, 1267 (2006).

[5] R. J. Glauber, Nucl. Phys. A774, 3 (2006).

[6] G. Goldhaber, W. B. Fowler, S. Goldhaber, and T. F. Hoang, Phys. Rev. Lett. 3, 181 (1959).

[7] G. Goldhaber, S. Goldhaber, W.-Y. Lee, and A. Pais, Phys. Rev. 120, 300 (1960).

[8] A. N. Makhlin and Y. M. Sinyukov, Z. Phys. C39, 69 (1988).

[9] T. Csörgő and B. Lörstad, Phys. Rev. C54, 1390 (1996).

[10] S. S. Adler et al., Phys. Rev. Lett. 93, 152302 (2004).

[11] S. Afanasiev et al., Phys.Rev.Lett. 103, 142301 (2009).

[12] A. Adare et al., 1709.05649.

[13] S. Afanasiev et al., Phys.Rev.Lett. 100, 232301 (2008).

[14] S. S. Adler et al., Phys. Rev. Lett. 98, 132301 (2007).

[15] R. Metzler, E. Barkai, and J. Klafter, Phys. Rev. Lett. 82, 3563 (1999).

[16] T. Csörgő, S. Hegyi, and W. A. Zajc, Eur. Phys. J. C36, 67 (2004). 

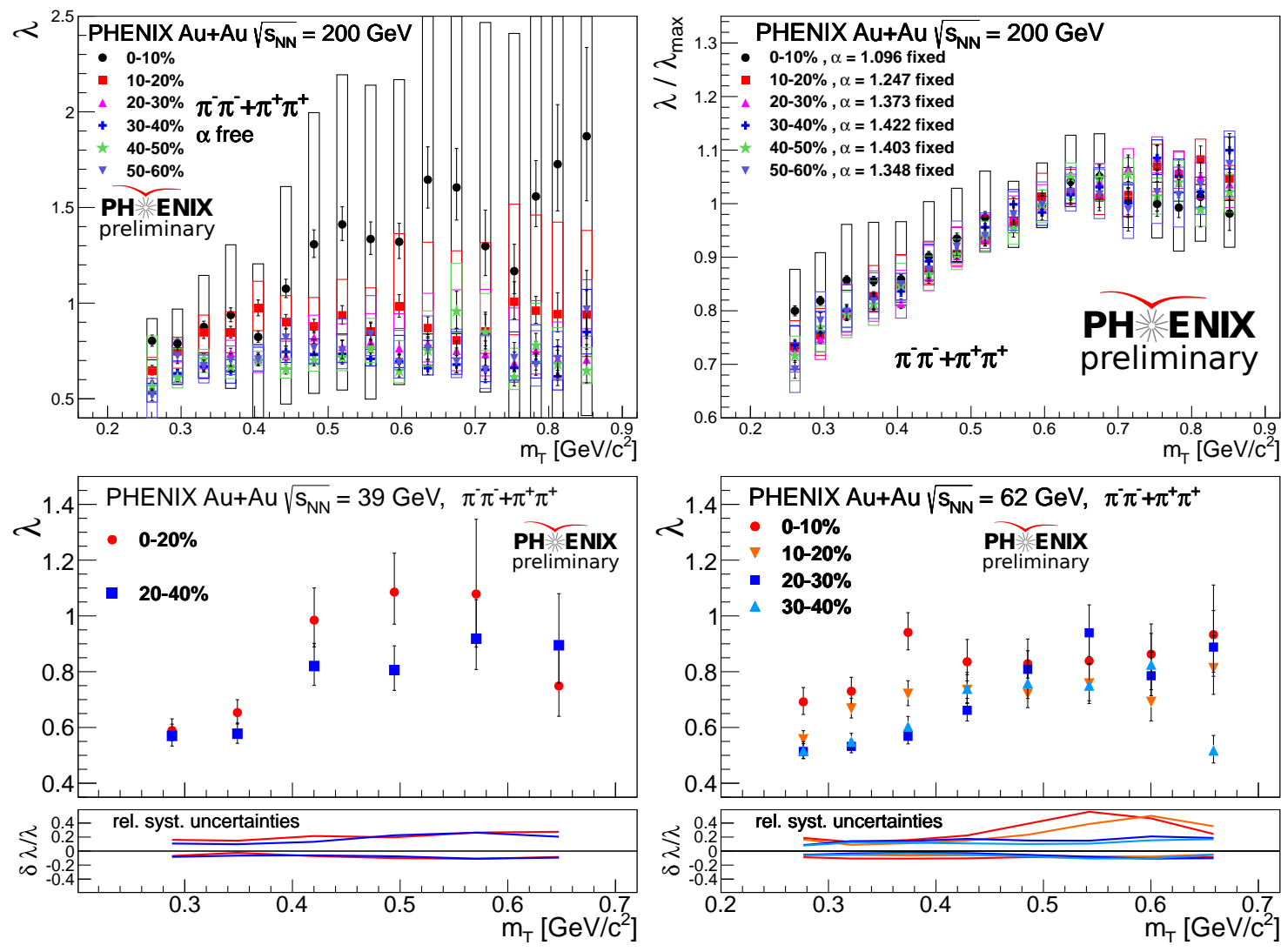

Figure 5: The fit parameter $\lambda$, for various centralities in $\sqrt{s_{N N}}=200 \mathrm{GeV} \mathrm{Au}+\mathrm{Au}$ collisions (top panels, free $\alpha$ on the left, fixed $\alpha$ fits on the right plot), and for lower collision energies (bottom panels). Statistical and systematic uncertainties are indicated similarly to Fig. 3.

[17] M. Csanád, T. Csörgő, and M. Nagy, Braz. J. Phys. 37, 1002 (2007).

[18] T. Csörgő, PoS HIGH-PTLHC08, 027 (2008).

[19] S. El-Showk et al., J. Stat. Phys. 157, 869 (2014).

[20] H. Rieger, Phys. Rev. B 52, 6659 (1995).

[21] M. A. Halasz et al., Phys. Rev. D58, 096007 (1998).

[22] M. A. Stephanov, K. Rajagopal, and E. V. Shuryak, Phys. Rev. Lett. 81, 4816 (1998).

[23] J. Bolz et al., Phys.Rev. D47, 3860 (1993).

[24] T. Csörgő, B. Lörstad, and J. Zimányi, Z. Phys. C71, 491 (1996).

[25] J. I. Kapusta, D. Kharzeev, and L. D. McLerran, Phys. Rev. D53, 5028 (1996).

[26] S. E. Vance, T. Csörgő, and D. Kharzeev, Phys. Rev. Lett. 81, 2205 (1998).

[27] T. Csörgő, R. Vértesi, and J. Sziklai, Phys.Rev.Lett. 105, 182301 (2010).

[28] M. Csanád, in 10th Bolyai-Gauss-Lobachevsky Conference on Non-Euclidean Geometry and its Applications (BGL-10) Gyöngyös, Mátra, Hungary, August 21-26, 2017 (MDPI, Basel, Switzerland, 2017). 

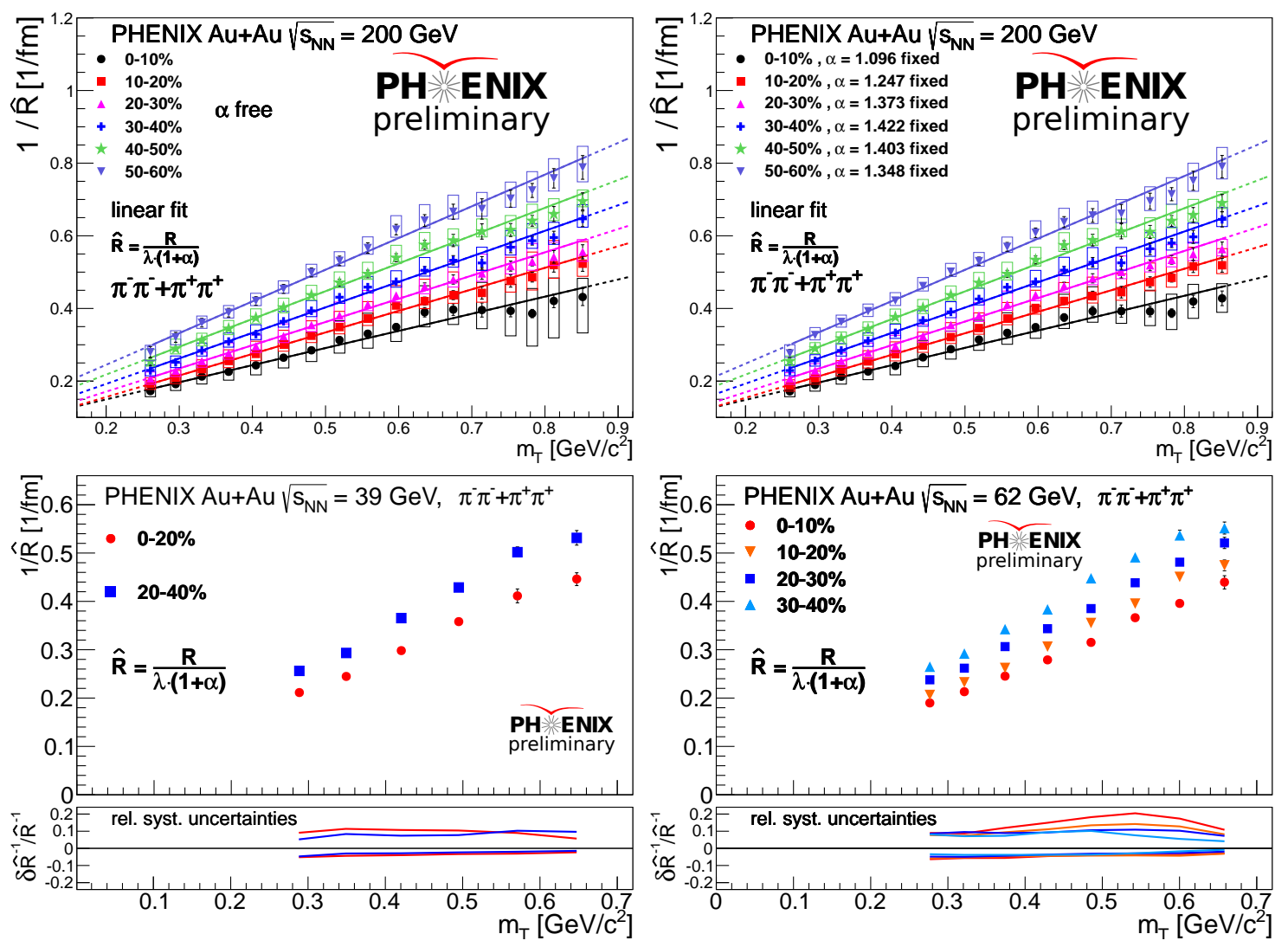

Figure 6: The fit parameter $R$, for various centralities in $\sqrt{s_{N N}}=200 \mathrm{GeV} \mathrm{Au}+\mathrm{Au}$ collisions (top panels, free $\alpha$ on the left, fixed $\alpha$ fits on the right plot), and for lower collision energies (bottom panels). Statistical and systematic uncertainties are indicated similarly to Fig. 3.

[29] D. Kincses, in 10th Bolyai-Gauss-Lobachevsky Conference on Non-Euclidean Geometry and its Applications (BGL-10) Gyöngyös, Mátra, Hungary, August 21-26, 2017 (MDPI, Basel, Switzerland, 2017). 